\title{
Evaluación de dos variedades de tomate: Chonto Santa Cruz y Río Grande (Lycopersicon esculentum Mill.) mediante técnicas de agricultura urbana en el municipio de Istmina, Chocó, Colombia
}

\author{
Evaluation of two tomato varieties: Chonto Santa Cruz and Río Grande \\ (Lycopersicon esculentum Mill.) using techniques of urban agriculture in the \\ municipality of Istmina, Chocó, Colombia
}

Mirla Perea Murillo*

\section{Resumen}

Se evaluó el rendimiento agronómico de dos variedades de tomate (Chonto Santa Cruz y Río Grande), mediante técnicas de agricultura urbana (cama y chorizo), utilizando para hidratación de las plantas agua y solución nutritiva compuesta de macro y micronutrientes. La investigación se realizó con el propósito de generar un paquete tecnológico de fácil adopción por las comunidades y contribuir al mejoramiento de la seguridad y soberanía alimentaria de las familias. Para esta investigación se utilizaron 12 camas y 12 chorizos; se analizaron variables a partir del efecto de tres factores: sistema de siembra, variedad y tipo de solución sobre la variable cuantitativa producción. Como resultado, se obtuvo que la variedad Río Grande cultivada en el sistema de siembra en cama, adicionándole solución nutritiva, presentó mayor rendimiento con una producción de 134 tomates que corresponden a 16.76 kilogramos a diferencia de la variedad Chonto Santa Cruz, que su producción fue de 78 tomates que corresponden a 1.7 kilogramos, utilizando el mismo sistema de siembra y de solución nutritiva. Todo esto teniendo en cuenta la tendencia actual de desarrollar mecanismos productivos que sean amigables con el medio ambiente, en una zona con suelos degradados por la actividad minera como el San Juan y la poca opción de consumir hortalizas frescas; este trabajo se convierte en una alternativa agrícola para las comunidades (madres cabeza de hogar, desplazados, niños y agricultores, entre otros).

Palabras clave: Diseño factorial, Macronutriente, Micronutriente, Seguridad alimentaria, Solución nutritiva.

\begin{abstract}
Agronomic performance of two varieties of tomato (Chonto Santa Cruz and Río Grande) by urban agriculture techniques (bed and chorizo) was assessed, using water hydration levels and nutrient solution composed of macro and micronutrients. The research was conducted with the purpose of generating a technology package easily adopted by communities and help improve the food security and sovereignty of families. For this investigation 12 beds and 12 sausages were used; variables were analyzed from the effect of three factors: planting system, variety and type of settlement on the quantitative variable production. As a result, it was found that the Río Grande variety grown in the bed planting system, add nutrient solution yielded better with production of tomatoes $134 \mathrm{~kg}$ corresponding to 16.76 unlike Chonto Santa
\end{abstract}

* Instituto de Investigaciones Ambientales del Pacífico (IIAP), Quibdó, Chocó. e-mail.mperea@iiap.org.co

Recibido: 23 de octubre de 2012

Aceptado: 1 de marzo de 2013 


\section{Bioetnia Volumen 10, 2013}

Cruz, its production was 78 tomatoes corresponding to 1.7 $\mathrm{kg}$, using the same planting system and nutrient solution. All this considering the current trend to develop production mechanisms that are friendly to the environment, in an area degraded by mining as the San Juan soils and little choice to consume fresh vegetables, this work becomes an alternative for agricultural communities (single mothers, displaced children, farmers, etc.).

Keywords: Factorial design, Food security, Macronutrient, Micronutrient, Nutrient solution.

\section{Introducción}

Como se ha venido planteando en los últimos tiempos sobre la necesidad de facilitar a las comunidades la producción de alimentos sanos que ayuden a mitigar los problemas de inseguridad alimentaria y desnutrición que padecen nuestros pueblos, la agricultura urbana se convierte en una estrategia adecuada para contribuir a mejorar esta situación, sobre todo para la producción de hortalizas como el tomate que pertenece a la familia de las Solanaceas (Buxadé et al. 2001).

Según la Asociación de Agrónomos Indígenas de Cañar (2003), el tomate es la hortaliza más cultivada en el mundo por su contenido nutricional y su demanda en la dieta diaria. Se lo puede cultivar a campo abierto y en invernadero, desde el nivel del mar hasta una altura de $3200 \mathrm{~m}$, es decir en zonas tropicales, valles y en zonas andinas en condiciones de invernadero. Esta especie se considera como una de las hortalizas más consumidas en el departamento del Chocó, sobre todo en el municipio de Istmina, pero poco cultivada en esta zona, afectando con esto la seguridad alimentaria de la comunidad y generando dependencia de este producto alimenticio hacia otras regiones del país.

Asimismo, se destaca la importancia de esta hortaliza por los diferentes usos, lo cual también depende de la variedad, tal es el caso del tomate Río Grande que se destaca por ser una de las variedades con mayor aceptación en el mercado y una de las variedades de tamaño grande, con un peso entre 90 y 120 gramos, presenta una forma ovalada, de porte mediano, tipo pera, color rojo vivo y piel fina, se caracteriza por presentar un hábito indeterminado y las plantas de esta variedad tienen una reducción profunda en su crecimiento a temperatura constante (Páez et al. 2000). Por otro lado, está el tomate Chonto Santa Cruz, otra de las variedades que más se comercializan, presenta crecimiento indeterminado, se adapta a zonas húmedas del trópico y sus frutos son de tamaño mediano con pesos menores de 100 gramos.

Este artículo pretende aportar elementos valiosos mediante el cultivo y evaluación de dos variedades de tomate
(Río Grande y Chonto Santa Cruz) en dos sistemas de siembra de agricultura urbana (cama y chorizo), utilizando dos tipos de nutrientes (solución nutritiva y agua), con el objeto de evaluar la adaptabilidad de estas variedades a las condiciones climáticas de la zona y utilizando estas técnicas de agricultura, porque esta última le permitiría a la población tener una mayor accesibilidad para consumir esta hortaliza y una menor dependencia por los productos extraregionales.

\section{Metodología}

Área de estudio. Se realizó en el barrio Cubis de la cabecera municipal de Istmina, ubicado astronómicamente a $05^{\circ} 09^{\prime}$ L, N y $76^{\circ} 41^{\prime}$ L, W, a una altura de $90 \mathrm{msnm}$, en el clima cálido húmedo, precipitación promedio anual superior a $11000 \mathrm{~mm}$, humedad relativa de $86 \%$, temperatura promedio de $28^{\circ}$ a $32^{\circ} \mathrm{C}$, corresponde a la zona de vida del bosque pluvial tropical (Figura 1). La principal actividad económica es la minería mecanizada, que ha originado el deterioro progresivo del suelo, seguido de la explotación maderera, el comercio y en baja escala la agricultura, la cual se realiza de manera tradicional, empleando pocos insumos agrícolas y escasa asistencia prestada al pequeño agricultor. Los principales sistemas agrícolas son: plátano, arroz, maíz, yuca y frutas (EOT 2005).

Métodos. Se empleó la metodología de agricultura urbana descrita por Marulanda (2005) donde se evaluó el rendimiento agronómico de dos variedades de tomate (Chonto Santa Cruz y Río Grande) mediante técnicas de agricultura urbana (cama y chorizo), utilizando dos tipos de soluciones (nutritiva y agua).

Fase de campo. En esta fase se elaboraron 36 germinadores, 18 para cada una de las variedades utilizando recipientes plásticos. Para acondicionar los germinadores se utilizó como sustrato arena fina, cascarilla de arroz y tierra de hormiga, en relación 1:1:1, se trazaron dos surcos en cada uno y se perforaron las bolsas con 12 hoyos a una profundidad de $1 \mathrm{~cm}$ sembrando en cada uno 3 semillas, para un total de 24 semillas por germinador, de acuerdo con las técnicas de agricultura urbanas ya mencionadas. A los 20 días se realizó el trasplante, se sembraron 8 plántulas de Lycopersicon esculentum de cada variedad.

Manejo agronómico del cultivo. Se realizó manejo agronómico según los requerimientos. El control de arvenses y plagas se efectuó manualmente y por último se realizó tutorado a las camas, para evitar el volcamiento de las plantas.

Fertilización. Para el caso de los sistemas cama y chorizo que requería fertilización, se efectuó diariamente utilizando soluciones nutritivas compuestas por macronutrientes (nitrógeno, fósforo y potasio) y micronutrientes (cobre, 


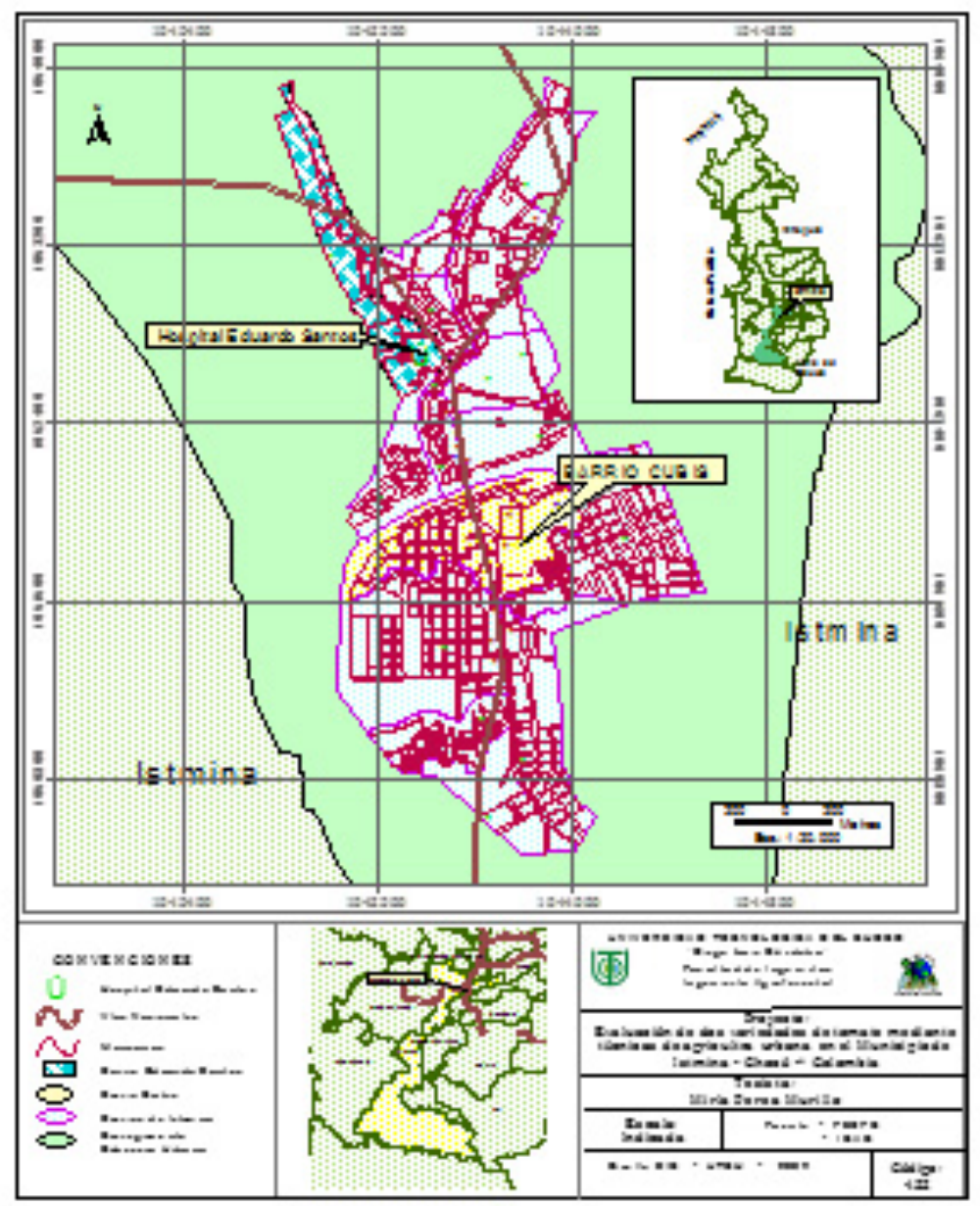

Figura 1. Ubicación del barrio Cubis en el municipio de Istmina.

boro, hierro, manganeso, zinc y molibdeno), suplementos que fueron adquiridos comercialmente. Durante la primera semana de germinación se adicionó $2.5 \mathrm{~cm}^{3}$ de macronutrientes y $1 \mathrm{~cm}^{3}$ de micronutrientes por cada litro de agua día por medio; de la segunda semana en adelante, se aplicó la dosis completa de los nutrientes $\left(5 \mathrm{~cm}^{3}\right.$ de macronutrientes y 2 de micronutrientes por cada litro de agua) diario. Un día de la semana se reemplazó la solución de nutriente por una cantidad mayor de agua (2-3 veces) de la cantidad que se había estado aplicando cada día, con el fin de lavar las sales que se van acumulando en el sustrato para evitar intoxicaciones o retrasos en el desarrollo del cultivo.

Etapa 2. Análisis estadístico. El diseño aplicado en esta investigación fue el factorial, porque se analizó el efecto de tres factores: sistema de siembra (cama y chorizo), la variedad (Río Grande y Santa Cruz), y el tipo de solución (agua y solución nutritiva "macro y micronutrientes" sobre la variable cuantitativa (producción).

En este experimento se estableció el efecto de tres factores (sistema de siembra, tipo de solución y el tipo de variedad) sobre la variable producción. Asimismo se determinó:

Si hubo diferencias entre las variedades y decidir cuál es la mejor.

- Si hubo diferencias entre los dos sistemas de siembra y y decidir cuál es el más adecuado.

- Si existieron diferencias entre los tipos de solución y decidir cual fue el más adecuada para lograr una mayor producción.

- Si el sistema de siembra y el tipo de variedad interaccionaron (es decir, si el efecto de uno de los factores dependió del nivel en el que se encontraba el otro). Ello significa contrastar si el efecto del sistema de siembra será distinto según la variedad.

La variable a medir fue la producción (cuántos tomates contados a partir de la siembra, medidos en kilogramos) mediante variables dependientes como: la variedad de tomates, el tipo de solución y el sistema de siembra. El procesamiento estadístico de los datos se realizó mediante el programa estadístico STARGRAPHIC, utilizandoANOVA para la comparación de las medias (Tabla 1). 
Bioetnia Volumen 10, 2013

Tabla 1

Diseño factorial

\begin{tabular}{|c|c|c|c|c|c|c|c|}
\hline \multicolumn{8}{|c|}{ Diseño del ensayo } \\
\hline \multicolumn{4}{|c|}{ Sistema de siembra en chorizo } & \multicolumn{4}{|c|}{ Sistema de siembra en cama } \\
\hline \multirow[b]{2}{*}{ Agua } & \multirow{2}{*}{$\begin{array}{r}\text { Variedad } 1 \\
\text { Nutriente }\end{array}$} & \multicolumn{2}{|c|}{ Variedad 2} & \multirow[b]{2}{*}{ Agua } & \multirow{2}{*}{$\begin{array}{c}\text { Variedad } 1 \\
\text { Nutriente }\end{array}$} & \multicolumn{2}{|c|}{ Variedad 2} \\
\hline & & Agua & Nutriente & & & Agua & Nutriente \\
\hline Chorizo & Chorizo & Chorizo & Chorizo & Cama & Cama & Cama & Cama \\
\hline Chorizo & Chorizo & Chorizo & Chorizo & Cama & Cama & Cama & Cama \\
\hline Chorizo & Chorizo & Chorizo & Chorizo & Cama & Cama & Cama & Cama \\
\hline
\end{tabular}

\section{Resultados y discusión}

\section{Fenología y rendimiento agronómico del cultivo \\ Fenología}

Germinación: la germinación se produjo para las dos variedades en un período de 4 a 6 días después de la siembra, lo cual coincide con lo expresado por Pérez et al. (2004) quien argumenta que este proceso se compone de tres etapas: absorción de agua, reposo y crecimiento, comprendido en un máximo de 5 días.

Floración: las plantas de L. esculentum de la variedad Río Grande, cultivadas en el sistema de agricultura urbana en cama, irrigadas con solución nutritiva, florecieron a los 49 días después del trasplante. Este resultado es similar al obtenido por Restrepo et al. (2008), quienes en su documento sobre fenología y floración del L. esculentum, argumentan que esta etapa se da entre 45 y 55 días y en chorizo a los 54 días. Las camas irrigadas con agua florecieron a los 52 días y en el sistema de siembra en chorizo a los 60 días después del trasplante.

Las plantas de L. esculentum de la variedad Chonto Santa Cruz, cultivadas en el sistema de agricultura urbana en cama, irrigadas con solución nutritiva, florecieron a los 50 días después del trasplante y en chorizo a los 55 días.

Las camas irrigadas con agua florecieron a los 55 días y en el sistema de siembra en chorizo a los 63 días después del trasplante. Las flores presentaron color amarillo, estilo y ovario simples, características morfológicas propias de esta especie.

Fructificación: las plantas de L. esculentum de la variedad Río Grande, cultivadas en el sistema de agricultura urbana en cama, irrigadas con solución nutritiva, fructificaron a los seis días después de la floración, y en chorizo a los nueve días. Las camas irrigadas con solo agua fructificaron a los ocho días y en el sistema de siembra en chorizo a los once días después de la floración.

Para la variedad Chonto Santa Cruz, cultivada en el sistema de siembra de agricultura urbana en cama, irrigadas con solución nutritiva fructificó a los siete días después de la floración y en chorizo a los 10 días y las camas irrigadas con solo agua fructificaron a los nueve días y en el sistema de siembra en chorizo a los doce días después de la floración.

Rendimiento agronómico. Se obtuvo que la variedad Río Grande sembrada en el sistema de siembra en cama irrigada con solución nutritiva, presentó una producción promedio de $16.7 \mathrm{~kg}$ y para el sistema de siembra chorizo fue de $8.5 \mathrm{~kg}$; asimismo, para el sistema de siembra en cama irrigada con agua, la producción fue de $15.6 \mathrm{~kg}$, a diferencia del sistema de siembra chorizo que fue de $7.6 \mathrm{~kg}$.

Para la variedad Chonto Santa Cruz sembrada en el sistema de siembra en cama irrigada con solución nutritiva, se presentó una producción promedio de $2.9 \mathrm{~kg}$ y para el sistema de siembra chorizo fue de $1.1 \mathrm{~kg}$; para el sistema de siembra en cama irrigada con agua, la producción fue también de $1.1 \mathrm{~kg}$.

Identificación de los insectos asociados con el cultivo. Se pudo identificar un insecto del orden Lepidóptero, familia Noctuidae, género Erynnis, especie ello asociado con el cultivo de L. esculentum, más conocido como gusano cachón. Este se presentó en la etapa de desarrollo del cultivo (entre trasplante hasta la floración). Este insecto no se consideró como plaga, porque no produjo daños significativos, sin embargo este insecto en estado larval forrajeó algunas hojas de las plantas de L. esculentum.

Por el tamaño que presenta esta larva se detectó fácil y el control se hizo de forma manual oportunamente. Puede ser a que en la mayoría de los casos relacionados con plagas la gravedad del daño depende de la tolerancia que el cultivador tenga con ellas porque si se hacen visitas frecuentes y se observan las plantas con detenimiento, se podrá detectar a tiempo la presencia de alguna de ellas y su eliminación se hará antes de que causen un perjuicio grande al cultivo (Marulanda 2005).

Análisis estadístico. Los supuestos del análisis de varianza, independencia, normalidad e igualdad de varianza 


\section{Evaluación de dos variedades de tomate. M Mosquera et al.}

Intera cciones y 95,0 Porcentajes Intervalos HSD de Tukey

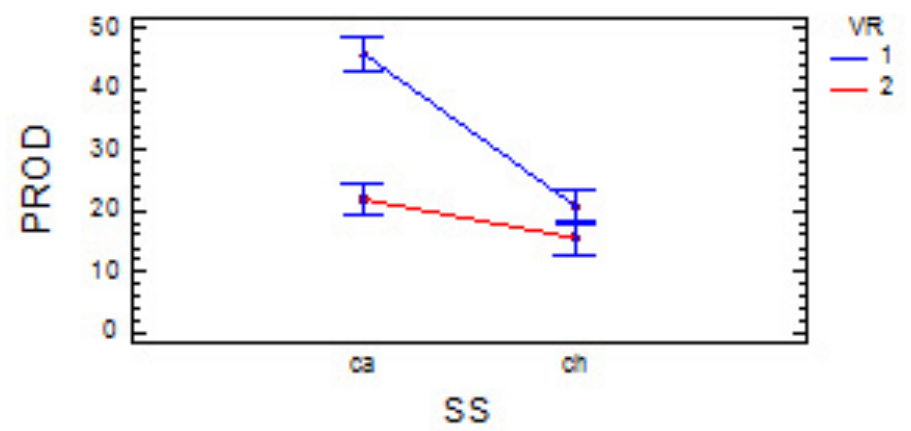

Figura 2. Interacción sistema de siembra-tipo variedad.

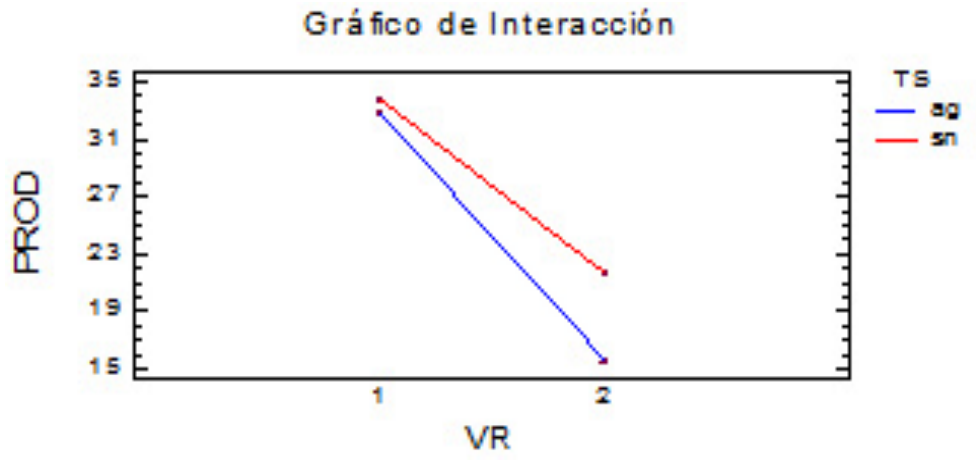

Figura 3. Interacción tipo de solución-tipo de variedad.

Medias y 95,0 Porcentajes Intervalos H SD de Tukey

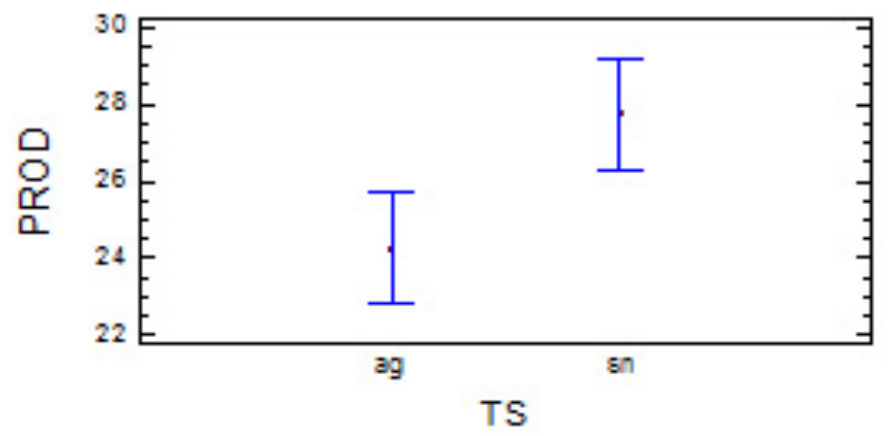

Figura 4. Medias de producción por tipo.

fueron verificados resultando aceptables.

La tabla del ANOVAnos indica que el tipo de solución=TS (agua $=$ ag, solución nutritiva $=\mathrm{Sn})$, sistema de siembra $=\mathrm{SS}(\mathrm{cama}=\mathrm{ca}$, chorizo $=\mathrm{ch})$ y el tipo de variedad=TV (Río Grande=variedad 1, Chonto Santa Cruz $=$ variedad 2), afectan significativamente a la variable producción (Figura 2). En relación con la producción se encontró que se obtienen los mejores resultados con el sistema de siembra cama y la variedad Río Grande, porque en las interacciones sistema de siembra-tipo variedad, se destaca que el sistema de siembra tiene un efecto reducido sobre la producción, cuando es empleado al sembrar la variedad Chonto Santa Cruz, pero un efecto positivo cuando se utiliza la variedad Río Gran- de. También se puede afirmar que el efecto de la variedad es pequeño cuando el sistema de siembra es chorizo y muy alto cuando el sistema de siembra empleado es cama.

Para el caso de la interacción tipo de solución-tipo de variedad, se afirma que se obtiene mejor resultado utilizando la variedad Río Grande, independientemente del tipo de solución empleada (Figura 3).

Por otro lado, se realizó un contraste sobre la igualdad de las medias para cada par de niveles de cada factor. La prueba utilizada en este caso, fue la de la diferencia significativa mínima (HSD) donde los análisis indican que existe diferencia estadísticamente significativa a un nivel de confianza del $95 \%$ entre las medias de producción de un tipo de sistema de siembra a otro, siendo el sistema cama con el que se logra el promedio más alto de producción de L. esculentum.

De igual manera, se destaca la diferencia entre las medias de producción de un tipo de solución a otro, siendo el tipo de solución nutritiva con la cual se logra mayor producción (Figura 4). Esto indica que el tratamiento utilizado con la solución nutritiva (macro y micronutriente) se constituye como un estimulador en los procesos biológicos de las plantas de $L$. esculentum, porque se corroboró que existe mayor producción con la utilización de este (Duarte et al. 2004).

En cuanto a la variedad, existen diferencias significativas entre las evaluadas, siendo mejor la variedad Río Grande, porque con ella se logra el promedio de producción más alto. Además tenemos con el cultivo de esta variedad plantas vigorosas que producen cosechas abundantes de L. esculentums en racimos hasta de $8 \mathrm{~cm}$ de diámetro con características organolépticas deseables por el productor $\mathrm{y} / \mathrm{o}$ consumidor (Thompson y Stockists 2005).

Igualmente, los resultados muestran que la producción más alta se logra al emplear sistema de siembra cama con solución nutritiva y la más baja cuando se emplea el sistema chorizo con solución agua y en cuanto al sistema de siembra según la variedad, la media más alta se logra al emplear el sistema de siembra cama para sembrar la variedad Río Grande y la más baja al sembrar la variedad Chonto Santa Cruz con sistema de siembra chorizo. 


\section{Bioetnia Volumen 10, 2013}

\section{Conclusiones}

El análisis e interpretación de los resultados obtenidos en este trabajo permite generar las siguientes conclusiones:

Se ha comprobado que la agricultura urbana constituye una actividad altamente productiva, sobre todo para familias del San Juan que a raíz de la crisis social se han visto obligadas a dejar sus tierras. Es una alternativa compatible con las labores del hogar, el estudio y las responsabilidades diarias de cada uno de los miembros de una familia, es una forma sencilla limpia y económica de hacer agricultura y su práctica no implica dedicación exclusiva en la inversión del tiempo, teniendo en cuenta que se exige un serio compromiso del cultivador para realizar las labores de manejo de la huerta de lo cual dependen los resultados exitosos.

Se tiene entonces que la producción varía según el tipo de solución, el sistema de siembra y la variedad empleada. Además existe interacción entre los factores sistema de siembra y tipo de variedad, o sea que la interacción es significativa.

El sistema de siembra de agricultura urbana que se debe implementar en el municipio de Istmina es el modelo cama, porque esta técnica le permite a los agricultores tener un mayor desarrollo de las plantas debido a que tiene la propiedad de retener los nutrientes que se encuentran en el sustrato.

Desde el punto de vista de las características agronómicas hay que destacar la capacidad de producción de la variedad Río Grande, porque esta presenta crecimiento determinado, no siempre requiere tutorado y el peso del fruto es mayor que el de la variedad Chonto Santa Cruz.

Desde el punto de vista de la conservación ambiental el tratamiento con la solución nutritiva resulta más atractivo para la agricultura, pues no contamina el medio ambiente y su uso de aplicación es excelente para un mayor rendimiento; esta aplicación de nutrientes tiene una connotación especial porque la función principal hoy en día es promover técnicas físicas no contaminantes que propicien aumentar la productividad agrícola.

En términos generales, se consigue una producción más alta bajo las condiciones climáticas del municipio de Istmina cuando se utiliza el sistema de siembra en cama, la variedad Río Grande y adicionándole la solución nutritiva compuesta por macro y micronutrientes.

\section{Literatura citada}

Asociación de agrónomos indígenas de Cañar (AAIC). 2003. El cultivo de tomate riñón en invernadero. Quito: AAIC.

Buxadé C, Curt D, Durán J, Fernández J, Linares P, Márquez L. 2001. Enciclopedia práctica de la agricultura y la ganadería. Barcelona: Editorial Océano. pp. 667-9.

Duarte C, Guevara G, Méndez M. 2004. Uso del agua activada y con tratamiento magnético del tomate en condiciones de organopónico. Rev Cien Tec Agropec. 13 (3): 1-15.

Esquema de Ordenamiento Territorial Istmina. 2005. Sistema de información y documentación municipal. Disponible en: http://cdim.esap.edu.co/ BancoConocimiento/I/istmina-choco-eot-2005

Marulanda H. 2005. Micro huertas familiares e hidroponía familiar, autogestión frente a la pobreza. Armenia: PNUD. pp. 63-84.

Páez A, Paz V, López J. 2000. Crecimiento y respuestas fisiológicas de plantas de tomate Río Grande en la época mayo-julio. Efecto del sombreado. Rev Fac Agron. 17 (2): 173-84.

Pérez J, Hurtado G, Aparicio V, Argueta Q, Larín M. 2004. Guía técnica Cultivo de Tomate. Centro Nacional de Tecnología Agropecuaria y Forestal. San Salvador: Editorial Corripio. pp. 215-33.

Restrepo E, Vallejo F, Lobo M. 2008. Fenología de la floración en tomate cultivado y especies silvestres relacionadas. Corporación Colombiana de Investigaciones Agropecuaria. 57 (2): 1-5.

Thompson M, Stockists M. 2005. Tomate pomodoro Río Grande. Boletín No 047. Group LTD Notice: Undefined variable. Disponible en: www/usr/ home/tandm/=includes/admin/admin.php4 on line 2217 ; tandm 